\title{
Tunable Flux Vortices in Two-Dimensional Dirac Superconductors
}

\section{Journal Article}

\section{Author(s):}

Zeytinoğlu, Sina; Imamoglu, Atac; Huber, Sebastian (D)

Publication date:

2020-05-22

Permanent link:

https://doi.org/10.3929/ethz-b-000416460

Rights / license:

In Copyright - Non-Commercial Use Permitted

Originally published in:

Physical Review Letters 124(20), https://doi.org/10.1103/physrevlett.124.207006

\section{Funding acknowledgement:}

671000 - Interacting polaritons in two-dimensional electron systems (EC)

771503 - Topological Mechanical Metamaterials (EC) 


\title{
Tunable Flux Vortices in Two-Dimensional Dirac Superconductors
}

\author{
Sina Zeytinoğlu $\odot,{ }^{1,2,3, *}$ Atac İmamoğlu, ${ }^{2}$ and Sebastian Huber ${ }^{1}$ \\ ${ }^{1}$ Institute for Theoretical Physics, ETH Zürich, CH-8093 Zurich, Switzerland \\ ${ }^{2}$ Institute for Quantum Electronics, ETH Zürich, CH-8093 Zurich, Switzerland \\ ${ }^{3}$ Department of Physics, Harvard University, Cambridge, Massachusetts, 02138, USA
}

(Received 17 May 2019; revised manuscript received 15 October 2019; accepted 24 February 2020; published 21 May 2020)

\begin{abstract}
The nontrivial geometry encoded in the quantum mechanical wave function has important consequences for both noninteracting and interacting systems. Yet, our understanding of the relationship between geometrical effects in noninteracting systems and their interacting counterparts is far from complete. Here, we demonstrate how the single-particle Berry curvature associated with the normal phase in two dimensions modifies the fluxoid quantization of a Bardeen-Cooper-Schrieffer superconductor. A discussion of the experimental scenarios where this anomalous quantization is expected is provided. Our work demonstrates the importance of variational Ansätze in making a clear connection between the Berry phases of single-particle and many-body wave functions.
\end{abstract}

DOI: 10.1103/PhysRevLett.124.207006

The realization that the Hilbert space of a quantum mechanical system can be endowed with a nontrivial geometry resulted in the discovery of a plethora of novel phenomena both in single- and many-particle quantum systems. In particular, the geometric considerations associated with single-particle wave functions have led to the initial discovery and eventual classification of noninteracting topological insulators (TIs) [1-4], as well as to the investigation of the properties associated with defects in these phases [5-8]. On the other hand, the fascinating physics arising from the interplay between topology and interactions between particles occupying nontrivial singleparticle states was investigated in the framework of fractional quantum Hall systems (FQHSs) $[9,10]$ and fractional Chern insulators (FCIs) $[11,12]$. However, establishing a direct relationship between the role of nontrivial geometry on the single- and the many-particle level has been difficult, due to the correlated nature of the latter.

In this Letter, we demonstrate a clear connection between the nontrivial geometry of a two-dimensional (2D) singleparticle band structure and the response properties of an associated Bardeen-Cooper-Schrieffer (BCS) superconductor [13]. In particular, we show that in a 2D system whose normal state is described by the massive Dirac model, the superconducting state obeys a modified fluxoid quantization condition, resulting in fractional flux vortices as well as in an unconventional Josephson response. It is important to emphasize that the superconducting state is not perturbatively related to the normal state. However, as we show below, the role of geometry on both sides of the phase transition is tractable using the BCS variational Ansatz.

The fluxoid quantization originates from the fact that deep inside a bulk superconductor, the overall phase of the order parameter is constant. In conventional superconductors, this overall phase has contributions from two sources: the external electromagnetic vector potential and the $U(1)$ phase of the order parameter. The fluxoid quantization can be understood as the total screening of the electromagnetic vector potential by the phase of the order parameter field [14]. However, as we show below, an additional contribution to the overall phase emerges for a BCS superconductor with an isotropic Fermi surface which encloses a nontrivial Berry flux $\phi_{B}$ [see Fig. 1(a)]. This additional contribution is related to the orbital currents in the superconductor and allows for screening of the (a)

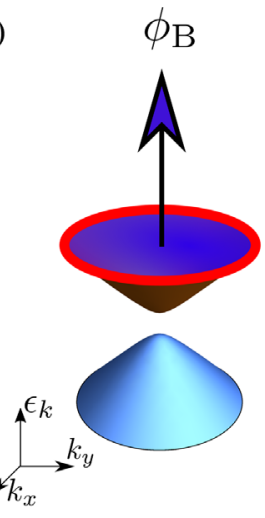

(b)

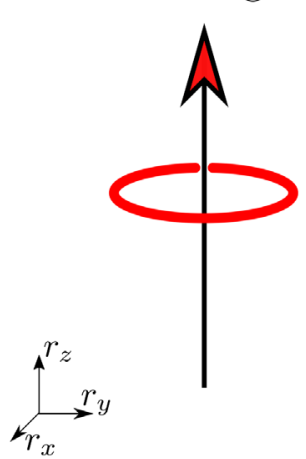

FIG. 1. (a) The fractional angular momentum of single particles on a circular Fermi surface enclosing nonzero Berry flux $\phi_{B}$. Similar to the magnetic field in real space, the Berry curvature carries an intrinsic angular momentum which is acquired by the single-particle states on the Fermi surface. The Berry flux $\phi_{B}$ enclosed in the Fermi surface determines the fractional part of the angular momentum associated with the states on the Fermi surface. (b) The fractional angular momentum due to the Berry flux can be considered as the momentum space analog of the anyon construction in Refs. [15,16]. 

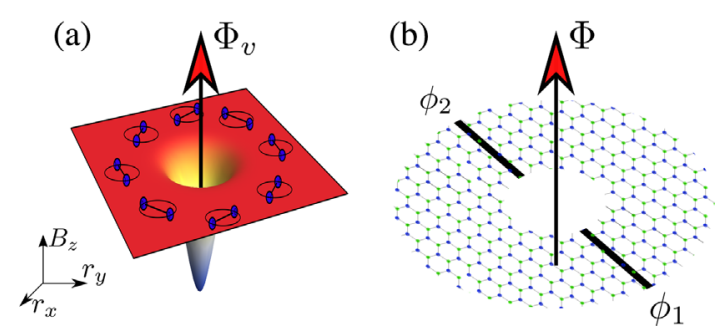

FIG. 2. The illustration of the configuration of the superconducting order parameter that stabilizes a vortex with fractional magnetic flux quantum $\Phi_{0}\left(\phi_{B} / \pi\right)$. The single valuedness of the order parameter is satisfied only if the relative coordinate of the Cooper pairs rotate by a multiple $q$ of $2 \pi$ around the vortex. Such a configuration realizes an adiabatic evolution of the order parameter as a function of the center of mass position. The Berry flux acquired by the order parameter contributes to the overall phase of the superconductor, modifying the conventional fluxoid quantization. (b) The modification of the fluxoid quantization also allows for building SQUIDs with tunable periodicity.

electromagnetic vector potential by the Aharanov-Bohm phase associated with the internal rotation of the Cooper pair. In particular, a $2 \pi$ rotation of the relative coordinate of the Cooper pair results in a change in the overall phase by $2 \phi_{B}$, allowing stable fractional flux vortices [see Fig. 2(a)].

Two-dimensional massive Dirac model is the simplest model with a nontrivial Berry flux enclosed by the Fermi surface and a rotationally symmetric energy dispersion. The Hamiltonian has the form

$$
H_{0}(k)=\left(f_{1, \mathbf{k}}^{\dagger} f_{2, \mathbf{k}}^{\dagger}\right)[\mathbf{d}(\mathbf{k}) \cdot \boldsymbol{\sigma}-\mu \rrbracket]\left(\begin{array}{c}
f_{1, \mathbf{k}} \\
f_{2, \mathbf{k}}
\end{array}\right)
$$

where $\sigma=\left(\sigma_{x}, \sigma_{y}, \sigma_{z}\right)$ is the vector of Pauli matrices, $\mathbb{1}$ is the identity matrix, and $f_{i, \mathbf{k}}^{\dagger}\left(f_{i, \mathbf{k}}\right)$ are the creation (annihilation) operators for particles with momentum $\mathbf{k}$ and spin or pseudospin label $i$. In the following, we let $|c(\mathbf{k})\rangle=$ $c_{\mathbf{k}}^{\dagger}|0\rangle \equiv\left(\alpha_{c, \mathbf{k}}^{(1)} f_{1, \mathbf{k}}+\alpha_{c, \mathbf{k}}^{(2)} f_{2, \mathbf{k}}\right)^{\dagger}|0\rangle$ and $|v(\mathbf{k})\rangle \equiv\left(\alpha_{v, \mathbf{k}}^{(1)} f_{1, \mathbf{k}}+\right.$ $\left.\alpha_{v, \mathbf{k}}^{(2)} f_{2, \mathbf{k}}\right)^{\dagger}|0\rangle$ denote the eigenvectors of $H_{0}(k)$, corresponding to conduction and valence bands, respectively. The single-particle Hamiltonian is parametrized by $\mathbf{d}(\mathbf{k})=\left(v_{F} k_{x}, v_{F} k_{y}, \delta\right)$, with (i) $2 \delta$ characterizing the minimum energy gap between the conduction and valence bands, (ii) the Fermi velocity $v_{F}$, which is taken to be unity for the rest of the Letter, and (iii) the chemical potential $\mu$, which we set to lie in the conduction band. We note that the single-particle Hamiltonian in Eq. (1) is a good effective low-energy description for a family of real materials, most notably transitional metal dichalcogenide (TMD) monolayers near the high symmetry $K$ points $[17,18]$, or surfaces of three-dimensional (3D) TIs in the proximity of a ferromagnetic insulator [19]. We give a more comprehensive list of experimental scenarios which can be modeled by Eq. (1) in our conclusion.
The massive Dirac model in Eq. (1) exhibits nontrivial geometry for both the conduction and valence band. The nontrivial geometry can be understood as a result of the Berry connection at momentum $\mathbf{k}$

$$
\mathbf{A}_{c}(\mathbf{k})=i\left\langle c(\mathbf{k}) \mid \nabla_{\mathbf{k}} c(\mathbf{k})\right\rangle=\frac{1}{k} \sin \left(\theta_{k} / 2\right)^{2} \mathbf{e}_{\phi},
$$

where $\theta_{k} \equiv \arccos \left(\delta / \sqrt{\delta^{2}+k^{2}}\right)$ and $\mathbf{e}_{\phi}$ is the unit vector in the azimuthal direction. The nontrivial connection results in a nonzero Berry flux $\phi_{B}$ enclosed in the conduction band Fermi surface

$$
\phi_{B}\left(k_{F}\right)=\oint_{\mathrm{FS}} d k \cdot \mathbf{A}_{c}(\mathbf{k})=\pi\left(1-\frac{\delta}{\sqrt{\delta^{2}+k_{F}^{2}}}\right),
$$

where $k_{F}$ denotes the Fermi momentum.

The normal state described by the Eq. (1) has a superconducting instability with respect to attractive interactions between electrons. In general, the interaction between the electrons can be written in second quantization in the (pseudo-) spin basis $(m, n \in\{1,2\})$ as

$H_{\text {int }}=\sum_{\mathbf{k}_{1}, \mathbf{k}_{2}, \mathbf{k}_{3}, \mathbf{k}_{4}, m, n} V_{\mathbf{k}_{1}, \mathbf{k}_{2}, \mathbf{k}_{3}, \mathbf{k}_{4}}^{n, m} f_{m, \mathbf{k}_{1}}^{\dagger} f_{n, \mathbf{k}_{2}}^{\dagger} f_{n, \mathbf{k}_{3}} f_{m, \mathbf{k}_{4}}$,

where $V_{\mathbf{k}_{1}, \mathbf{k}_{2}, \mathbf{k}_{3}, \mathbf{k}_{4}}^{n, m}<0$ for all $\left\{\mathbf{k}_{i}\right\}$, and $V_{\mathbf{k}_{1}, \mathbf{k}_{2}, \mathbf{k}_{3}, \mathbf{k}_{4}}^{n, m} \propto$ $\delta_{\mathbf{k}_{1}+\mathbf{k}_{2}, \mathbf{k}_{3}+\mathbf{k}_{4}}$ ensures momentum conservation.

For BCS superconductivity, the only relevant degrees of freedom are the zero momentum Cooper pairs consisting of electrons on the conduction band Fermi surface [13]. Hence, we consider only the terms where $\mathbf{k}_{1}=-\mathbf{k}_{2}$ and $\mathbf{k}_{3}=-\mathbf{k}_{4}$ in Eq. (4). Projecting the interaction Hamiltonian onto the conduction band, we obtain

$$
\tilde{H}_{\text {int }}=\sum_{\mathbf{k}, \mathbf{k}^{\prime}} U_{\mathbf{k}, \mathbf{k}^{\prime}} c_{\mathbf{k}}^{\dagger} c_{-\mathbf{k}}^{\dagger} c_{\mathbf{k}^{\prime}} c_{-\mathbf{k}^{\prime}},
$$

where the strength of projected interactions is

$$
U_{\mathbf{k}, \mathbf{k}^{\prime}}=\sum_{n, m} V_{\mathbf{k},-\mathbf{k}, \mathbf{k}^{\prime},-\mathbf{k}^{\prime}}^{n, m} \alpha_{c \mathbf{k}}^{(n) *} \alpha_{c-\mathbf{k}}^{(m) *} \alpha_{c \mathbf{k}^{\prime}}^{(m)} \alpha_{c-\mathbf{k}^{\prime}}^{(n)}
$$

In the context of our work, the most remarkable feature of the projected interaction in Eq. (5) is that when we restrict the momenta on the Fermi surface (i.e., $\mathbf{k}=k_{F} \hat{\mathbf{k}}$, and we denote the azimuthal angle of $\hat{\mathbf{k}}$ by $\left.\phi_{\hat{\mathbf{k}}}\right)$, the coefficients $U_{k_{F}}^{(l)}$ of the partial-wave expansion, defined through $U_{k_{F} \hat{\mathbf{k}}, k_{F} \hat{\mathbf{k}}^{\prime}}=\sum_{l} U_{k_{F}}^{(l)} e^{i l\left(\phi_{\hat{\mathbf{k}}}-\phi_{\hat{\mathbf{k}}^{\prime}}\right)}$, are redistributed with respect to the coefficients $V_{k_{F}}^{(l)}$ of the bare interaction. For example, in the massive Dirac model in Eq. (1), and $s$ wave interactions in the orbital basis $V^{(l)} \propto \delta_{l, 0}$, the partialwave coefficients of the interaction projected onto the 
conduction band is purely $p$ wave. In particular, $U_{k_{F}}^{(l)} \propto \delta_{l, 1}$, with

$$
U_{k_{F}}^{(1)}=\left(V_{k_{F}}^{12}\right)^{(0)}\left(1-\frac{\phi_{B}}{2 \pi}\right) \frac{\phi_{B}}{2 \pi},
$$

where we used the constraints $\left(V_{k_{F}}^{n n}\right)^{(l)}=0$ and $U_{k_{F}}^{(l)}=0$ for even $l$ arising from the fermionic anticommutation relations obeyed by operators $f_{m, \mathbf{k}}, f_{m, \mathbf{k}}^{\dagger}$ and $c_{\mathbf{k}}, c_{\mathbf{k}}^{\dagger}$, respectively. In general, when the bare interaction potential has multiple partial-wave components, the coefficients $U_{k_{R}}^{(l)}$ obey

$$
\begin{aligned}
U_{k_{F}}^{(l)}= & \left(V_{k_{F}}^{11}\right)^{(l)}\left(1-\frac{\phi_{B}}{2 \pi}\right)^{2}+\left(V_{k_{F}}^{12}\right)^{(l-1)}\left(1-\frac{\phi_{B}}{2 \pi}\right) \frac{\phi_{B}}{2 \pi} \\
& +\left(V_{k_{F}}^{22}\right)^{(l-2)} \frac{\phi_{B}^{2}}{2 \pi} .
\end{aligned}
$$

The redistribution of the partial-wave components can be understood as a direct consequence of the nontrivial Berry phase inclosed in the single-particle Fermi surface. Equation (7) expresses an effect that is similar to the emergence of effective $p$-wave pairing from $s$-wave interactions, as studied in TI surfaces [20] proximity coupled to an $s$-wave superconductor, as well as in the spectroscopic $[21,22]$ and transport [23] signatures of Berry curvature in 2D excitons. Furthermore, in three dimensions, the above discussion leads to the prediction of nodal superconductivity in Weyl semimetals $[24,25]$.

Next, we treat the superconducting phase where the Cooper pairs consist of electrons on the conduction band Fermi surface which encloses a Berry flux of $\phi_{B}\left(k_{F}\right)$. In the weak coupling regime, we can describe the superconducting ground state using the BCS Ansatz

$$
|\mathrm{BCS}\rangle=\prod_{k}\left(u_{\mathbf{k}}+v_{\mathbf{k}} c_{\mathbf{k}}^{\dagger} c_{-\mathbf{k}}^{\dagger}\right)|0\rangle,
$$

where $|0\rangle$ is the electronic vacuum of the conduction band. The BCS Ansatz parametrizes the expectation value of the Cooper pair $\left\langle\mathrm{BCS}\left|c_{\mathbf{k}} c_{-\mathbf{k}}\right| \mathrm{BCS}\right\rangle=u_{\mathbf{k}}^{*} v_{\mathbf{k}} \equiv F_{\mathbf{k}}$. Given the BCS gap equation $\Delta_{\mathbf{k}}=\sum_{\mathbf{k}^{\prime}} U_{\mathbf{k k}^{\prime}} F_{\mathbf{k}^{\prime}}$, the expectation value $F_{\mathbf{k}}$ can be written as $\left|F_{\mathbf{k}}\right| e^{i l_{0} \phi_{\hat{\mathbf{k}}}}$, where $l_{0}$ is odd and maximizes $\left|U_{k_{F}}^{l}\right|$.

Naively, one could conclude from the above discussion that the expectation value of the many-body angular momentum operator is $\hbar l_{0} n_{c}$, where we defined the number of condensed pairs as $n_{c} \equiv \sum_{\mathbf{k}} F_{\mathbf{k}}$. However, a more careful calculation shows that this conclusion is incorrect in the presence of the nontrivial Berry curvature. The expectation value of the relative angular momentum operator is (see Supplemental Material [26]) [27]

$$
\begin{aligned}
\left\langle L_{\mathrm{rel}}\right\rangle & \equiv-\frac{1}{2}\left\langle\mathrm{BCS}\left|\sum_{i \neq j}\left(\hat{r}_{i}-\hat{r}_{j}\right) \times\left(\hat{p}_{i}-\hat{p}_{j}\right)\right| \mathrm{BCS}\right\rangle \\
& =\hbar \sum_{\mathbf{k}} F^{*}(\mathbf{k})\left(-i \partial_{\phi}\right) F(\mathbf{k})+\sum_{\mathbf{k}} F^{*}(\mathbf{k}) \hbar \frac{\phi_{B}\left(k_{F}\right)}{\pi} F(\mathbf{k}) \\
& =n_{c} \hbar\left(l_{0}+\frac{\phi_{B}\left(k_{F}\right)}{\pi}\right) .
\end{aligned}
$$

We observe that the expectation value of the angular momentum operator is shifted with respect to the naive guess above by twice the angular momentum associated with $\phi_{B}\left(k_{F}\right)$ in Eq. (3). Indeed, the Berry flux contribution can be traced back to the fact that the second quantized position operator projected onto the conduction band is $[10,28]$

$$
\begin{aligned}
\hat{r} & =\sum_{\mathbf{k}, \mathbf{k}^{\prime}}\left\langle c\left(\mathbf{k}^{\prime}\right)|\hat{r}| c(\mathbf{k})\right\rangle c_{\mathbf{k}^{\prime}}^{\dagger} c_{\mathbf{k}} \\
& =\sum_{\mathbf{k}, \mathbf{k}^{\prime}}\left[-i \partial_{\mathbf{k}}+A_{c}(\mathbf{k})\right] \delta_{\mathbf{k}, \mathbf{k}^{\prime}} c_{\mathbf{k}^{\prime}}^{\dagger} c_{\mathbf{k}} .
\end{aligned}
$$

Equivalently, the angular momentum of the Cooper pair is given by the eigenvalue of the covariant derivative in momentum space, $D_{\phi}=-i \partial_{\phi}+\left[2 \phi_{B}\left(k_{F}\right) / 2 \pi\right]$.

This result can be intuitively understood by considering the analogy between the single-particle wave functions on the Fermi surface enclosing the Berry flux $\phi_{B}$, and the wave functions of a particle on a ring which encloses a magnetic flux $\Phi_{\text {Mag }}$ (see Fig. 1). As discussed in Refs. [15,16], in both cases, the eigenfunctions have angular momenta whose values are shifted with respect to their conventional integer values by a fraction associated with $\phi_{B}$ or $\Phi_{\mathrm{Mag}}$. Thus, the unconventional expectation value of the Cooper pair angular momentum can be understood as the sum of the shifted angular momenta of their constituent particles.

What are the physical effects of the fractional angular momentum expectation value of the Cooper pairs? We show that one interesting consequence of the fractional angular momentum is its effect on the orbital currents. We calculate the orbital current contribution in the Ginzburg Landau (GL) free energy from the microscopic model using the functional integral formalism (Supplemental Material [26]) $[29,30]$. To this end, we consider a configuration of the order parameter where the relative orientation of the Cooper pairs is adiabatically rotated as a function of their center of mass position $R$ [see Fig. 2(a)]. As a result, the gradient term in the GL free energy density can be shown to be

$$
\begin{aligned}
F_{\text {grad }}= & \frac{\hbar^{2} \rho_{s}}{2 m^{*}}\left(-2 \frac{e}{\hbar} \mathbf{A}_{\text {e.m. }}(R)\right. \\
& \left.+\nabla \theta(R)+\left(l_{0}+\frac{\phi_{B}}{\pi}\right) \nabla \phi_{k}(R)\right)^{2},
\end{aligned}
$$


where $\rho_{s} \equiv|\Delta|^{2}$ is the superfluid density for an isotropic superconductor, $m^{*}$ is the effective mass of the Dirac electron, $\mathbf{A}_{\text {e.m. }}(R)$ is the electromagnetic vector potential, and $\theta(R)$ is the $U(1)$ phase of the order parameter. We omit the $k_{F}$ dependence of the Berry flux for simplicity. Taking the variation of the free energy with respect to $\mathbf{A}_{\text {e.m. }}$, we find that the supercurrent including the effect of a variation in the relative orientation of the Cooper pair is

$$
\begin{aligned}
\mathbf{J}_{s}=\frac{\delta F}{\delta \mathbf{A}_{\mathrm{e} . \mathrm{m} .}}= & \frac{\hbar^{2} \rho_{s}}{m^{*}}\left[-2 \frac{e}{\hbar} \mathbf{A}_{\mathrm{e} . \mathrm{m} .}(R)\right. \\
& \left.+\nabla \theta(R)+\left(l_{0}+\frac{\phi_{B}}{\pi}\right) \nabla \phi_{k}(R)\right] .
\end{aligned}
$$

While the first and the second term in the above expression can be attributed to the charge and mass currents in a conventional superconductor, respectively, the third term is attributed to the orbital currents and contains the effect of the Berry flux enclosed in the Fermi surface. We note that because the normal state has a rotationally symmetric (isotropic) Fermi surface, the energy associated with the orbital currents goes to zero for configurations where the wavelength of the variation is taken to infinity.

The form of the orbital current contribution in Eq. (13) modifies the possible vortex states of the superconductor. As is well known, the magnetic field inside a superconductor is screened due to the Meissner effect [29,31], resulting in the condition that away from the vortex core, the supercurrents should vanish. This statement is captured by the following equation:

$$
\oint_{C} d \mathbf{l} \cdot \mathbf{J}_{s}=0
$$

where $C$ denotes a path around the vortex core, and we assumed that the distance between any point on $C$ and the vortex core is much larger than the effective London penetration length [32,33] of the 2D superconductor. Inserting Eq. (13) into Eq. (14), we find the minimum value of the magnetic flux $\Phi_{v} \equiv \oint_{C} d l \cdot \mathbf{A}_{\text {e.m. }}$. the vortex can accommodate to be

$$
\Phi_{v}=\frac{2 \pi \hbar}{2 e}\left[p+\left(l_{0}+\phi_{B} / \pi\right) q\right],
$$

where $p, q \in \mathbb{Z}$ denote the winding numbers of the $U(1)$ phase and the orientation angle $\phi_{\mathbf{k}}$ of the Cooper pairs along the contour, respectively. The minimal magnetic flux $\Phi_{\min }$ enclosed in the vortex core in a superconductor on a band with nontrivial Berry curvature is given by setting $q=1$ and $p=-l_{0}$ :

$$
\Phi_{\min }=\frac{h}{2 e} \phi_{B} / \pi \equiv \Phi_{0} \phi_{B} / \pi
$$

Given that $2 \phi_{B}$ can be interpreted as the Aharanov-Bohm phase acquired by the Cooper pair wave function as the orientation of the pair is adiabatically rotated by $2 \pi$, the configuration with $q=1$ corresponds to a vortex with fractional flux quantum $\Phi_{\min }$ that is stable due the screening of the electromagnetic field by the Aharanov-Bohm phase [see Fig. 2(a)].

The modification of the flux quantization in the presence of nontrivial Berry curvature and an isotropic Fermi surface also bears interesting consequences for a superconducting quantum interference device (SQUID) [see Fig. 2(b)]. The total current $i_{T}$ through the SQUID consisting of two Josephson junctions is

$i_{T}=i_{1}+i_{2}=2 I_{c} \cos \left(\frac{\phi_{1}-\phi_{2}}{2}\right) \sin \left(\frac{\phi_{1}+\phi_{2}}{2}\right)$,

where $I_{c}$ is the critical current of each junction and $\phi_{j}$ denotes the phase difference between the two sides of the $j$ th Josephson junction. When the thickness of the superconducting regions connecting the two Josephson junctions is much larger then the penetration length, we can define a contour $C$ for which Eq. (14) is satisfied. Then the relation between the phase difference $\phi_{1}-\phi_{2}$ and the total flux $\Phi$ is

$$
\frac{\Phi}{\Phi_{0}}=\frac{\phi_{1}-\phi_{2}}{2 \pi}+p+\left(l_{0}+\phi_{B} / \pi\right) q,
$$

where the winding numbers $p, q \in \mathbb{Z}$ have the same meaning as in Eq. (15). Inserting Eqs. (18) into (17), and setting $l_{0}=p=0$ for simplicity, the total current simplifies to

$i_{T}=2 I_{c} \cos \left(\phi_{B} q+\pi \frac{\Phi}{\Phi_{0}}\right) \sin \left(\phi_{1}+\phi_{B} q-\pi \frac{\Phi}{\Phi_{0}}\right)$.

Maximizing the driving current with respect to $\phi_{1}$, we obtain

$$
i_{\max } \approx 2 I_{c}\left|\cos \left(\phi_{B} q+\pi \frac{\Phi_{\mathrm{ext}}}{\Phi_{0}}\right)\right|,
$$

where we neglected the self-inductance of the superconducting loop (i.e., $\Phi=\Phi_{\text {ext }}$ ). The value of the winding number $q$ in Eq. (20) is given by the configuration which minimizes the kinetic energy of the system for a given $\Phi_{\text {ext }}$. As a result, the Josephson current through the SQUID has a periodicity that is a fraction of the conventional SQUID.

Before we conclude, we discuss possible experimental realizations of the physics presented above. The experimental setups where the single-particle Hamiltonian in Eq. (1) can be realized include (i) surface modes of a 3D TI in the proximity of a ferromagnetic insulator $[19,20]$, (ii) an isotropic anomalous Hall system [34], (iii) a single $K$ valley 
of a TMD monolayer [18,35], and (iv) a single $K$ valley of a bilayer graphene biased by an in-plane electric field [36].

The effects discussed above will also be present if each electron comprising the Cooper pair can be described by a separate massive Dirac Hamiltonian with the same Berry flux enclosed by each Fermi surface. Such systems include (v) a graphene monolayer on a hexagonal antiferromagnetic substrate [37] and (vi) a cold atom realization of a weakly doped Chern insulator [38,39].

For systems (i), (ii), and (v) above, the effects discussed above can be in principle observed by inducing a superconducting gap via proximity effect to a conventional $s$-wave superconductor. However, this strategy is not viable for systems which do not break time-reversal (TR) symmetry [(iii) and (iv)] since the proximity effect only allows pairing between zero total momentum Cooper pairs, and the $K$ and $-K$ valleys in (iii) and (iv) carry opposite Berry curvature. Consequently, the superconducting gap in (iii) and (iv) should be opened via intrinsic attractive [40] or repulsive [41] interactions which favor intravalley pairing. Lastly, in cold atom settings (vi), reliable Feshbach resonances allow greater freedom to realize pairing with desired properties. Once the desired superconductivity is achieved, the biggest challenge in observing the proposed effect is to find systems where the Dirac particles have a small mass compared to the chemical potential. From this point of view, a platform based on the surface modes of a 3D TI is the strongest candidate amongst those listed above.

In conclusion, we have demonstrated a direct relation between a nontrivial single-particle Berry phase enclosed in an isotropic Fermi surface and the modification of the fluxoid quantization in the associated BCS superconductor. A natural extension of our treatment is to include the effects of anisotropy and disorder. We also emphasize that our approach to combine the projected many-body position operators and with a mean field Ansatz can be applied to other weakly correlated systems consisting of excitons [23] or polarons [42].

We acknowledge useful discussions with Manfred Sigrist, Dima Geshkenbein, Aline Ramires, Ovidiu Cotlet, Mark Fisher, and Tomas Bzdusek, and Jose Luis Lado. This work is supported by a European Research Council (ERC) Advanced investigator grant (POLTDES) and Consolidator grant (TopMechMat). S.Z. gratefully acknowledges financial support from the Swiss National Science Foundation through Grant No. P2EZP2_184320.

*Corresponding author. sina_zeytinoglu@fas.harvard.edu

[1] A. P. Schnyder, S. Ryu, A. Furusaki, and A, W. W. Ludwig, Classification of topological insulators and superconductors, AIP Conf. Proc. 1134, 10 (2009).
[2] A. Kitaev, Periodic table for topological insulators and superconductors, AIP Conf. Proc. 1134, 22 (2009).

[3] L. Fu, Topological Crystalline Insulators, Phys. Rev. Lett. 106, 106802 (2011).

[4] B. Bradlyn, L. Elcoro, J. Cano, M. G. Vergniory, Z. Wang, C. Felser, M. I. Aroyo, and B. A. Bernevig, Topological quantum chemistry, Nature (London) 547, 298 (2017).

[5] R. Jackiw and C. Rebbi, Solitons with fermion number 1/2, Phys. Rev. D 13, 3398 (1976).

[6] W. P. Su, J. R. Schrieffer, and A. J. Heeger, Solitons in Polyacetylene, Phys. Rev. Lett. 42, 1698 (1979).

[7] D. A. Ivanov, Non-Abelian Statistics of Half-Quantum Vortices in $p$-Wave Superconductors, Phys. Rev. Lett. 86, 268 (2001).

[8] J.C. Y. Teo and T.L. Hughes, Topological defects in symmetry-protected topological phases, Annu. Rev. Condens. Matter Phys. 8, 211 (2017).

[9] R. B. Laughlin, Anomalous Quantum Hall Effect: An Incompressible Quantum Fluid with Fractionally Charged Excitations, Phys. Rev. Lett. 50, 1395 (1983).

[10] S. M. Girvin, The quantum Hall effect: Novel excitations and broken symmetries, in Aspects Topologiques de la Physique en Basse Dimension. Topological Aspects of Low Dimensional Systems (Springer-Verlag, Berlin and Les Editions de Physique, Les Ulis, 2000), pp. 53-175.

[11] N. Regnault and B. A. Bernevig, Fractional Chern Insulator, Phys. Rev. X 1, 021014 (2011).

[12] T. Neupert, L. Santos, C. Chamon, and C. Mudry, Fractional Quantum Hall States at Zero Magnetic Field, Phys. Rev. Lett. 106, 236804 (2011).

[13] J. Bardeen, L. N. Cooper, and J. R. Schrieffer, Theory of superconductivity, Phys. Rev. 108, 1175 (1957).

[14] M. Tinkham, Introduction to Superconductivity: Second Edition, Dover Books on Physics (Dover Publications, New York, 2004).

[15] F. Wilczek, Quantum Mechanics of Fractional-Spin Particles, Phys. Rev. Lett. 49, 957 (1982).

[16] F. Wilczek and M. Flux, Angular Momentum, and Statistics, Phys. Rev. Lett. 48, 1144 (1982).

[17] Q. H. Wang, K. Kalantar-Zadeh, A. Kis, J. N. Coleman, and M.S. Strano, Electronics and optoelectronics of twodimensional transition metal dichalcogenides, Nat. Nanotechnol. 7, 699 (2012).

[18] X. Xu, W. Yao, D. Xiao, and T.F. Heinz, Spin and pseudospins in layered transition metal dichalcogenides, Nat. Phys. 10, 343 (2014).

[19] Y. L. Chen, J.-H. Chu, J. G. Analytis, Z. K. Liu, K. Igarashi, H.-H. Kuo, X. L. Qi, S.-K. Mo, R. G. Moore, D. H. Lu et al., Massive dirac fermion on the surface of a magnetically doped topological insulator, Science 329, 659 (2010).

[20] L. Fu and C. L. Kane, Superconducting Proximity Effect and Majorana Fermions at the Surface of a Topological Insulator, Phys. Rev. Lett. 100, 096407 (2008).

[21] A. Srivastava and A. Imamoğlu, Signatures of BlochBand Geometry on Excitons: Nonhydrogenic Spectra in Transition-Metal Dichalcogenides, Phys. Rev. Lett. 115, 166802 (2015).

[22] J. Zhou, W.-Y. Shan, W. Yao, and D. Xiao, Berry Phase Modification to the Energy Spectrum of Excitons, Phys. Rev. Lett. 115, 166803 (2015). 
[23] W. Yao and Q. Niu, Berry Phase Effect on the Exciton Transport and on the Exciton Bose-Einstein Condensate, Phys. Rev. Lett. 101, 106401 (2008).

[24] S. Murakami and N. Nagaosa, Berry Phase in Magnetic Superconductors, Phys. Rev. Lett. 90, 057002 (2003).

[25] Y. Li and F. D. M. Haldane, Topological Nodal Cooper Pairing in Doped Weyl Metals, Phys. Rev. Lett. 120, 067003 (2018).

[26] See Supplemental Material at http://link.aps.org/supplemental/ 10.1103/PhysRevLett.124.207006 for detailed derivation of the orbital currents and the expectation value of the Cooper pair orbital angular momentum. We also give an introductory discussion on the Pearl vortex in 2D superconductors.

[27] A. J. Leggett, A theoretical description of the new phases of liquid ${ }^{3} \mathrm{He}$, Rev. Mod. Phys. 47, 331 (1975).

[28] E. I. Blount, Formalisms of band theory, Solid State Phys. 13, 305 (1962).

[29] A. Altland and B. D. Simons, Condensed Matter Field Theory (Cambridge University Press, New York, 2010).

[30] C. Mudry, Lecture Notes on Field Theory in Condensed Matter Physics (World Scientific, Hackensack, 2014).

[31] T. P. Orlando and K. A. Delin, Foundations of Applied Superconductivity (Addison-Wesley, Reading, MA, 1991), Vol. 8.

[32] J. Pearl, Current distribution in superconducting films carrying quantized fluxoids, Appl. Phys. Lett. 5, 65 (1964).

[33] A. L. Fetter, Flux penetration in a thin superconducting disk, Phys. Rev. B 22, 1200 (1980).

[34] N. Nagaosa, J. Sinova, S. Onoda, A. H. MacDonald, and N. P. Ong, Anomalous Hall effect, Rev. Mod. Phys. 82, 1539 (2010).
[35] A. Srivastava, M. Sidler, A. V. Allain, D. S. Lembke, A. Kis, and A. Imamoğlu, Valley Zeeman effect in elementary optical excitations of monolayer WSe2, Nat. Phys. 11, 141 (2015).

[36] J. B. Oostinga, H. B. Heersche, X. Liu, A. F. Morpurgo, and L. M. K. Vandersypen, Gate-induced insulating state in bilayer graphene devices, Nat. Mater. 7, 151 (2008).

[37] Z. Qiao, W. Ren, H. Chen, L. Bellaiche, Z. Zhang, A. H. MacDonald, and Q. Niu, Quantum Anomalous Hall Effect in Graphene Proximity Coupled to an Antiferromagnetic Insulator, Phys. Rev. Lett. 112, 116404 (2014).

[38] M. Aidelsburger, M. Lohse, C. Schweizer, M. Atala, J. T. Barreiro, S. Nascimbene, N. R. Cooper, I. Bloch, and N. Goldman, Measuring the Chern number of Hofstadter bands with ultracold bosonic atoms, Nat. Phys. 11, 162 (2015).

[39] G. Jotzu, M. Messer, R. Desbuquois, M. Lebrat, T. Uehlinger, D. Greif, and T. Esslinger, Experimental realization of the topological Haldane model with ultracold fermions, Nature (London) 515, 237 (2014).

[40] M. A. Rahimi, A. G. Moghaddam, C. Dykstra, M. Governale, and U. Zülicke, Unconventional superconductivity from magnetism in transition-metal dichalcogenides, Phys. Rev. B 95, 104515 (2017).

[41] Y.-T. Hsu, A. Vaezi, M.H. Fischer, and E.-A. Kim, Topological superconductivity in monolayer transition metal dichalcogenides, Nat. Commun. 8, 14985 (2017).

[42] M. Sidler, P. Back, O. Cotlet, A. Srivastava, T. Fink, M. Kroner, E. Demler, and A. Imamoglu, Fermi polaronpolaritons in charge-tunable atomically thin semiconductors, Nat. Phys. 13, 255 (2017). 